\title{
Enjeux d'éducation aux changements climatiques auprès des communautés
}

Liette Vasseur

\section{(2) OpenEdition}

Journals

Édition électronique

URL : http://journals.openedition.org/ere/5908

DOI : $10.4000 /$ ere. 5908

ISSN : 2561-2271

Éditeur

Centr'ERE

Référence électronique

Liette Vasseur, "Enjeux d'éducation aux changements climatiques auprès des communautés », Éducation relative à l'environnement [En ligne], Volume 15 - 2 | 2020, mis en ligne le 15 novembre 2020, consulté le 22 février 2021. URL : http://journals.openedition.org/ere/5908 ; DOI : https://doi.org/ 10.4000/ere.5908 


\title{
Enjeux d'éducation aux changements climatiques auprès des communautés
}

\author{
Liette Vasseur
}

1 Éduquer devient un défi majeur pour les convaincre les populations de la nécessité d'entreprendre des actions visant à atténuer les changements climatiques tout en s'adaptant à ces modifications (Pruneau et coll., 2008). Cependant, les phénomènes associés aux changements climatiques ne sont pas faciles à comprendre. Cela demande en tout premier lieu une certaine compréhension de notions de physique, de géographie et de météorologie. Malheureusement, celles-ci ne sont pas nécessairement enseignées à l'école. Plusieurs de ces notions ne sont traitées que dans des cours avancés ou spécialisés de la fin du secondaire ou des études supérieures. Étant donné sa complexité, la question des changements climatiques n'est pas toujours abordée ou elle l'est superficiellement, car le personnel enseignant reste souvent réticent à l'introduire par manque de formation à ce sujet ou de peur de ne pouvoir répondre aux questions des élèves (Pruneau et coll. 2006). Cela veut dire que peu d'adultes et de jeunes ont une bonne compréhension des changements climatiques.

2 L'adaptation se définit ici comme un ajustement ou une action qui permet de réduire l'exposition aux risques provenant d'un phénomène d'origine climatique comme par exemple les inondations causées par une tempête pluviale (Intergovernmental Panel on Climate Change, 2007). Initialement, on a cru que les stratégies d'adaptation d'ordre technologique ou relevant de certaines pratiques sociales seraient adéquates. Cependant, ces premiers efforts d'adaptation n'ont pas abouti au succès et à l'avancée que les pays et les gouvernements escomptaient. Cela peut être relié au manque d'information et d'éducation concernant les changements climatiques au sein de la population.

3 En raison de ces limites et du besoin de mieux intégrer la compréhension des dynamiques environnementales dans les stratégies d'adaptation, d'autres approches ont été promues, la plupart étant basées sur la prise en compte des processus naturels. 
On reconnait maintenant que les solutions basées sur la nature auraient avantage à être adoptées dans toutes les sphères de décision, car elles permettent de réduire l'empreinte écologique des humains. Elles font appel à une diversité d'actions, d'ordre environnemental, social ou économique. Parmi ces solutions, l'approche de l'adaptation basée sur les écosystèmes $(\mathrm{AbE})$ apparaît avantageuse pour réduire la vulnérabilité des populations locales aux changements climatiques. L'AbE se définit comme toute action ou stratégie qui permet de promouvoir la biodiversité ainsi que la conservation et la réhabilitation des écosystèmes naturels comme bases pour réduire les impacts des changements climatiques (Andrade Perez et coll., 2010). Cette approche est endossée par plusieurs organisations internationales comme l'Union internationale de la conservation de la nature (UICN), les organismes des Nations unies, dont le Programme Environnement, et certains gouvernements des pays de l'Union européenne. Un élément important de l'AbE est la promotion de la gouvernance écosystémique basée sur «les interactions entre les structures, processus et traditions qui déterminent comment le pouvoir et les responsabilités sont exercés. Comment les décisions sont prises et comment les citoyens et autres acteurs peuvent intervenir dans la gestion des ressources naturelles, incluant la conservation de la biodiversité » (Graham et coll., 2003, p. 2). Un point commun entre l'AbE et la gouvernance écosystémique est l'implication et la participation active des communautés locales. Ces communautés doivent donc d'abord comprendre les enjeux régionaux des changements climatiques.

Cet article a pour objectifs d'expliquer d'abord l'AbE et la gouvernance écosystémique pour ensuite discuter de l'importance de l'éducation et surtout de l'apprentissage social, afin de susciter des actions locales concrètes. Cette discussion s'appuie sur des études de cas passés ou en cours, où ces approches ont été ou sont utilisées pour promouvoir l'adaptation aux changements climatiques, en même temps que le développement viable. L'AbE diffère d'autres processus d'adaptation qui sont basés davantage sur les solutions uniquement sociales et technologiques. La prochaine section traitera des raisons qui incitent à privilégier l'AbE et la gouvernance écosystémique.

\section{Pourquoi adopter une approche écosystémique?}

5 Au cours de la dernière décennie, l'approche écosystémique a pris de l'ampleur en raison de l'augmentation des défis environnementaux auxquels nous faisons face. Bien que cette approche ait été promue par plusieurs organisations internationales depuis longtemps, la plupart des décideurs gouvernementaux et des corporations ont toujours cru que les technologies pourraient résoudre tous nos problèmes (Keulartz, 2012). Toutefois, les données récentes montrent à quel point cela n'est pas le cas et les problèmes sont de plus en plus complexes et difficiles à cerner, tant au niveau de leur amplitude que des solutions possibles (Vasseur et coll., 2017).

6 L'approche d'adaptation basée sur les écosystèmes reconnaît qu'un écosystème en santé et résilient remplit des fonctions essentielles et fournit des services écosystémiques efficaces, permettant la survie des humains. Un écosystème en santé est normalement plus diversifié et complexe dans sa structure, ce qui lui permet de résister et même de se régénérer efficacement à la suite d'événements extrêmes comme des pluies torrentielles ou des tempêtes majeures. Par sa diversité, l'écosystème peut exercer plusieurs fonctions complémentaires et certaines d'entre elles peuvent en 
soutenir d'autres lorsque celles-ci sont affectées par un événement climatique. Depuis 2000, avec le projet d'Évaluation des écosystèmes pour le millénaire ${ }^{1} »$ (MEA, 2005), les services écosystémiques ont été de plus en plus mis en lumière. Avec la mise en place de la Plateforme intergouvernementale scientifique et politique sur la biodiversité et les services écosystémiques (IPBES) ${ }^{2}$, le concept est aussi de plus en plus présent dans les médias et fait l'objet de recherches en sciences de l'environnement. Les services écosystémiques sont les contributions que l'on reçoit de la nature et qui nous permettent de survivre et de croître socialement et économiquement (Diaz et coll., 2018). Ces services incluent ceux de l'approvisionnement, du support, de la régulation et de l'apport culturel. Les services d'approvisionnement comprennent par exemple, la production de nourriture, l'eau potable, le bois pour les meubles ou le papier. Les services de support et de régulation, quant à eux, comprennent les cycles des éléments nutritifs, la formation des sols, la régulation du climat, etc. Finalement, l'apport culturel est rattaché aux besoins non matériels (bien que plusieurs peuvent être calculés financièrement) tels que l'esthétique, les loisirs et la méditation (MEA, 2005 ; Diaz et coll., 2018).

7 Plusieurs études ont montré l'importance des services écosystémiques pour les communautés locales. C'est le cas des palétuviers dans les écosystèmes de mangroves, comme zones tampons contre les tempêtes. Leur santé permet aux populations locales adjacentes d'être moins impactées lors de tempêtes majeures. Il a été observé par exemple, qu'une zone de palétuviers en santé peut réduire le nombre de morts en cas d'événement climatique (Ahammad et coll., 2013). L'AbE est donc directement reliée au développement viable, car elle permet d'un côté de générer ou d'assurer des bénéfices socio-économiques (comme pour les mangroves, la pêche aux crevettes et aux crabes, et la production de bois) et de l'autre, de protéger contre les tempêtes. La destruction des mangroves pour des bassins d'élevage commercial de crevettes à grande échelle ou pour des développements résidentiels (et il en va de même pour les marais salés et les zones inondables près des rivières) détruit cette zone tampon et augmente significativement les probabilités de conséquences graves pour les communautés côtières. Il est donc clair que l'AbE est devenue une stratégie essentielle pour plusieurs communautés, surtout celles qui dépendent des ressources naturelles pour leur développement et leur survie. Pour y arriver, l'AbE se base sur la communauté locale.

\section{Un ingrédient important : la communauté locale}

8 L'AbE et la gouvernance écosystémique se basent sur l'action locale et donc sur la communauté comme point de départ, bien que ces approches puissent convenir également à plus grande échelle. L'une des conditions essentielles est la prise en compte des conditions locales et culturelles. Le but principal de cet ancrage local des actions et des décisions est de réduire le plus possible les vulnérabilités sociales et environnementales. L'AbE se base sur la confiance, l'inclusion, la participation active et l'engagement de la communauté. Cela requiert des décideurs et de tous les membres de la communauté une grande transparence et une imputabilité de leurs décisions et leurs actions. Toutes les institutions et les partenaires communautaires doivent être impliqués. Cette gouvernance doit aussi être adaptative, c'est-à-dire que les décisions, les stratégies ou les activités entreprises peuvent être modifiées selon les suivis et les réponses du système social et écologique en question. L'AbE vise le maintien des bénéfices produits par les services écosystémiques. Elle s'appuie sur des programmes 
d'éducation aux changements climatiques pour tous les âges et cultures (Zölch et coll., 2018).

En effet, la participation est essentielle comme le montrent fréquemment les analyses de projets d'AbE (Harvey et coll., 2017). Mais cette participation ne peut avoir lieu si les acteurs ont une faible compréhension des enjeux. L'éducation est à la base de tout projet qui se veut participatif et qui requiert l'engagement de tous les membres de la communauté. Or ceux-ci ne se situent pas tous au même niveau économique, cognitif ou de croyances. Les personnes plus influentes peuvent avoir une éducation qui leur permet de comprendre davantage les enjeux, mais cela n'est pas toujours le cas. Le fait que les notions complexes reliées aux changements climatiques soient mal comprises contribue à la perception trop répandue que les individus ne peuvent rien faire et que tout peut être résolu au niveau gouvernemental. Par ailleurs, il reste trop souvent au sein de la population des personnes qui nient les changements climatiques et ce, pour plusieurs raisons, incluant le manque d'éducation. Il est donc important de débuter des projets d'adaptation aux changements climatiques en évaluant le niveau d'information et d'éducation de la communauté et de s'assurer de fournir d'abord les connaissances nécessaires.

\section{Apprendre ensemble}

L'éducation environnementale et plus précisément l'éducation aux changements climatiques peut se faire à tous les niveaux, de façon formelle ou informelle. Pour intégrer des concepts comme l'adaptation basée sur les écosystèmes, il faut d'abord assurer une compréhension des changements climatiques. Sans une bonne appréciation des risques et aléas liés à ces phénomènes, ainsi que de la valeur des services écosystémiques pour la viabilité des communautés, il est difficile en effet de faire comprendre le concept d'adaptation. L'éducation est donc essentielle pour favoriser l'appropriation des connaissances de base et convaincre tous les membres de la communauté de l'importance de l'AbE et de les inciter à s'engager en ce sens.

11 Mais qui sont ces membres? Comment les engager dans ce processus? Dans le cas du projet «Défis des communautés côtières à l'heure des changements climatiques (DCC) ", un projet du programme Alliance de recherche université-communauté financé par le Conseil de recherche en sciences humaines du Canada (Vasseur et coll., 2018), plusieurs stratégies ont favorisé l'apprentissage social et la participation. Initialement, il a été fort utile de dresser un portrait des dix communautés côtières ciblées par le projet, de façon à mieux identifier les groupes d'intérêt composant la population et de n'oublier personne. Cette étape a été importante pour bien comprendre entre autres le niveau d'éducation des membres de chacune des communautés

Par la suite, en fonction des enjeux de compréhension du phénomène des changements climatiques au sein des communautés, une diversité de stratégies participatives ont pu être utilisées. Par exemple, des assemblées de cuisine, des ateliers de formation, des cafés science et des camps de jeunes ont été offerts. Le but de recourir à diverses stratégies était de s'assurer que l'information était bien communiquée et partagée pour améliorer les connaissances et ce, à tous les niveaux. Un tel apprentissage social permet de croiser les connaissances scientifiques et les savoirs traditionnels ou locaux. Cet apport de connaissances de terrain peut aider à situer les enjeux dans le contexte de la communauté et ainsi à co-développer des solutions qui sont plus viables, car elles 
rejoignent plus de gens. En effet, les échanges entre les connaissances scientifiques et les savoirs locaux et traditionnels peuvent aider les gens à mieux comprendre les enjeux et mener à l'adoption de solutions efficaces qui respectent la culture des gens (Adger et coll., 2013).

Plusieurs de ces stratégies sont donc participatives et peuvent s'adapter au niveau d'éducation de la communauté. Elles sont flexibles et ne requièrent pas toujours l'accès à une salle de rencontre. Par exemple, dans le cas d'un autre large projet au NouveauBrunswick, une initiative a été menée auprès la communauté Mikmaq d'Elsipogtog. Il s'agissait de la conservation des plantes médicinales traditionnelles qui se trouvaient dans les marais salés et qui étaient menacées par les changements climatiques, plus spécifiquement, par l'augmentation du niveau de la mer et par les tempêtes. Les chercheures et les gens de la communauté ont travaillé ensemble sur le terrain pour d'abord inventorier les endroits où les plantes se trouvaient. L'éducation communautaire s'est appuyée sur des échanges de connaissances en utilisant des cercles de paroles (Vasseur et Tremblay, 2014). Cette approche non formelle a été très efficace pour cette communauté qui a pu par la suite soumettre un nouveau projet par elle-même pour continuer d'autres travaux sur les changements climatiques et la santé de la population.

Une leçon apprise de ces divers projets est l'importance de transmettre les connaissances de façon à ce qu'elles soient faciles à comprendre. Dans le cas de la communauté de San Juan dans le Chimborazo en Équateur, la communauté a exprimé dès le départ un besoin d'éducation environnementale sur les changements climatiques. Cette communauté marginalisée, l'une des plus pauvres du Chimborazo, est composée à $87 \%$ de Kichwas. Comme le profil de cette population l'a montré (Mur et coll., en préparation), le niveau d'éducation est très limité, de même que l'accès à l'information. Cependant, les membres de la communauté s'inquiétaient, car, dans les Andes, la température augmente et les précipitations ont diminué de près de $30 \%$, causant des sécheresses et une réduction du débit d'eau pour l'Amazone équatoriale. Les activités économiques de cette communauté, principalement basées sur l'agriculture, étaient compromises. Les connaissances des habitants au sujet des enjeux passaient surtout par leurs perceptions des changements. Le partage de leurs connaissances initiales, complétées par des connaissances scientifiques, a pu aider à les engager dans l'action, en développant leurs capacités à trouver des solutions viables (Kupika et coll., 2019).

D'autres stratégies d'éducation non formelle peuvent être utilisées dans certains contextes, comme la production de sites web où les informations et connaissances sont transmises de façon à interagir avec les gens de la communauté. Dans notre étude de cas de la ville de Lincoln (www.brockul.ca/unesco-chair), l'approche utilisée est celle de la production de cartes interactives géographiques et évolutives de la côte du lac Ontario afin de sensibiliser la population aux dangers de se construire trop près du lac.

\section{Conclusion}

L'intégration de l'AbE dans les politiques publiques et les activités d'une communauté ne peut se réaliser sans une éducation de la population, qu'elle soit formelle ou non formelle. Pour que des solutions appropriées soient adoptées et implantées, il faut que les gens de la communauté comprennent les enjeux et aient assez de compétences pour 
agir. Cependant, l'éducation ne sera pas le seul ingrédient pour que l'AbE soit effectivement réalisée. Comme le souligne Wamsler (2015), les communautés sont complexes et ce, surtout en ce qui concerne leur gouvernance, d'où l'importance de promouvoir une gouvernance écosystémique pour améliorer les possibilités de succès de l'AbE. Au niveau individuel, il faut aussi reconnaitre que si l'apport d'informations peut aider grandement à mieux comprendre les enjeux des changements climatiques, les valeurs, les attitudes et les comportements des personnes peuvent ne pas changer à cause de diverses pressions sociales, comme celle de l'attrait de certains styles de vie (Vasseur et Pickering, 2015; Meira et González Gaudiano, 2016). Comprendre les interrelations entre le savoir, les comportements et les actions des gens reste complexe et requiert davantage d'études et de réflexion. Comme le mentionnent Pruneau et coll. (2008), on parle ici d'une éducation à la complexité.

\section{BIBLIOGRAPHIE}

Adger, W. N., Barnett, J., Brown, K., Marshall, N. et O'brien, K. (2013). Cultural dimensions of climate change impacts and adaptation. Nature Climate Change, 3 (2), 112-117.

Ahammad, R., Nandy, P. et Husnain, P. (2013). Unlocking ecosystem based adaptation opportunities in coastal Bangladesh. Journal of Coastal Conservation, 17, 833-840.

Andrade Pérez, A., Herrera Fernández, B. et Cazzolla Gatti, R. (eds.). (2010). Climate change and ecosystems : case-studies of impacts and Ecosystem-based Adaptation. IUCN Commission on Ecosystem Management (CEM), Climate Change Adaptation Group.

Diaz, S., Pascual, U., Stenseke, M., Martín-López, M., Watson, R. et coll. (2018). Assessing nature's contributions to people. Science, 359, 270-272.

Graham, J., Amos, B. et T. Plumptre. (2003). Governance principles for protected areas in the $21^{\text {st }}$ century. Prepared for The Fifth World Parks Congress Durban, South Africa.

Harvey, C.A., Martínez-Rodrígueza, M.R., Cárdenas, J.M., Avelino, J., Rapidel, B., Vignola, R., Donatti C.I. et Vilchez-Mendoza, S. (2017). The use of Ecosystem-based Adaptation practices by smallholder farmers in Central America. Agriculture, Ecosystems and Environment, 246, 279-290.

Intergovernmental Panel on Climate Change (IPCC). (2007). Climate change 2007 : mitigation of climate change. Dans B. Metz, O.R. Davidson, P.R. Bosch, R. Dave, L.A. Meyers (eds.) Contribution of working group III to the fourth assessment report of the intergovernmental panel on climate change., Cambridge : Cambridge University Press

Keulartz, J. (2012). The emergence of enlightened anthropocentrism in ecological restoration. Nature and Culture, 7, 48-71.

Kupika, O.L., Gandiwa, E., Nhamo G. et Kativu, S. (2019). Local ecological knowledge on climate change and Ecosystem-Based Adaptation strategies promote resilience in the Middle Zambezi Biosphere Reserve, Zimbabwe. Scientifica. 
Meira, P. et González Gaudiano, E.J. (2016). Les défis éducatifs du changement climatique : La pertinence de la dimension sociale. Éducation relative à l'environnement 13 (2). Repéré à http:// journals.openedition.org/ere/730

Millennium Ecosystem Assessment (MEA). (2005). Ecosystems and human well-being : Synthesis. Washington, DC : Island Press.

Pruneau D., Doyon A. Langis J, Vasseur L., Ouellet E., Martin G., McLaughlin E. et Boudreau G. (2006). The process of change experimented by teachers and students when voluntarily trying environmental behaviors. Applied Environmental Education and Communication, 5 (1), 33-40.

Pruneau, D., Demers, M. et Khattabi, A. (2008). Éduquer et communiquer en matière de changements climatiques : défis et possibilités. [Vertigo] La revue électronique en sciences de l'environnement, 8 (2).

Vasseur, L. et Tremblay, E. (2014). Coastal ecosystem in Kouchibouguac National Park of Canada : Adaptation possibilities for protecting traditional knowledge of local a community. Dans C. Buyck, (ed.). Safe Havens : Protected areas for disaster risk reduction and climate change adaptation (p. 33-40). Gland, Switzerland : IUCN.

Vasseur, L. et Pickering, G. (2015). Feeding the social animal : how to engage Canadians in climate change mitigation. Dans C. Potvin (ed.). Acting on climate change : Extending the dialogue among Canadians. 163-170.

Vasseur, L., Horning, D., Thornbush, M., Cohen-Shacham, E., Andrade, A., Barrow, E., Edwards, S., Wit, P. et Jones, M. (2017). Complex problems and unchallenged solutions : Bringing ecosystem governance to the forefront of the UN Sustainable Development Goals. Ambio doi :10.1007/ s13280-017-0918-6

Vasseur, L., Thornbush, M. et Plante, S. (2018). Climate change adaptation to coastal storms in Atlantic Canada. SpringerBrief in Geography. Cham, Switzerland : Springer Nature.

Wamsler, C. (2015). Mainstreaming ecosystem-based adaptation transformation toward sustainability in urbangovernance and planning. Ecology and Society, 20 (2), 30.

Zölch, T., Wamsler, C. et Pauleit, S. (2018). Integrating the ecosystem-based approach into municipal climate adaptation strategies : The case of Germany. Journal of Cleaner Production, 170, 966-977.

\section{NOTES}

1. www.millenniumassessment.org/fr/About.html

www.canada.ca/fr/environnement-changement-climatique/organisation/affairesinternationales/partenariats-organisations/biodiversite-services-ecosystemiques-plateformeintergouvernementale.html 


\section{RÉSUMÉS}

Bien que les acteurs locaux soient les premiers à subir les conséquences des changements climatiques, il ne leur est pas toujours facile de comprendre les phénomènes en cause et de faire des choix d'actions pertinents. En se basant sur des études menées au Canada et en Équateur, cet article montre à quel point il est important de connaître les besoins de formation des différentes communautés et d'adopter des approches et stratégies éducatives appropriées. Certaines approches permettent non seulement d'informer, mais aussi de favoriser l'apprentissage social, d'éclairer la prise de décision et de stimuler l'engagement. En particulier, nous proposons une approche d'adaptation aux changements climatiques basée sur les écosystèmes et la gouvernance écosystémique. Les stratégies éducatives que nous avons associées à une telle approche incluent la vulgarisation des connaissances scientifiques, la participation active des citoyens permettant le partage des savoirs locaux et le développement d'outil de visualisation sur le web.

Connecting actions promoting sustainable development and climate change adaptation to local people is not always easy despite the fact that local people are the first to be impacted. It remains a challenge to have people understand these issues at the local level. Based on work in Canada and Ecuador, this article demonstrates the importance to first understand what knowledge is needed by the members of the communities and then knowing how to transfer this knowledge. Certain tools allow to not only educate but also help to take charge of the decision-making process through engagement and social learning. The approaches described here are the ecosystem-based adaptation to climate change and ecosystem governance. Education at all levels includes the mobilization of scientific knowledge, the active participation of citizens to share local knowledge, and the development of visualization tool on the web.

\section{INDEX}

Mots-clés : apprentissage social, approche écosystémique, gouvernance, changements climatiques, vulgarisation des connaissances, adaptation basée sur les écosystèmes

Keywords : social learning, ecosystem-based approach, governance, climate change, adaptation, knowledge translation

\section{AUTEUR}

\section{LIETTE VASSEUR}

Liette Vasseur est professeure titulaire au département des sciences biologiques de l'Université Brock à Saint Catharines, en Ontario, au Canada. Elle est membre du Centre de recherche sur la durabilité de l'environnement. Courriel : lvasseur [@] brocku.ca 